PROCEEDINGS OF THE

AMERICAN MATHEMATICAL SOCIETY

Volume 129, Number 8 , Pages 2205-2207

S 0002-9939(00)05815-9

Article electronically published on December 28, 2000

\title{
ON THE NUMBER OF MAXIMAL SUM-FREE SETS
}

\author{
TOMASZ ŁUCZAK AND TOMASZ SCHOEN
}

(Communicated by John R. Stembridge)

\begin{abstract}
It is shown that the set $\{1,2, \ldots, n\}$ contains at most $2^{n / 2-2^{-28}} n$ maximal sum-free subsets, provided $n$ is large enough.
\end{abstract}

A set $A \subseteq[n]=\{1,2, \ldots, n\}$ is sum-free if for any two elements $a, b \in A$ we have $a+b \notin A$. A sum-free set $A \subseteq[n]$ is maximal if it is not contained in any other sum-free subset of $[n]$. Let $s(n)$ and $s_{\max }(n)$ denote the number of sum-free and maximal sum-free subsets of $[n]$, respectively. Since the set of odd numbers is sum-free, and so is each of its subsets, $s(n) \geq 2^{\lceil n / 2\rceil}$. It is conjectured that, in fact, we have $s(n) \leq c 2^{n / 2}$ for some constant $c>0$ but at this moment we know only (see Calkin [2] and Alon [1]) that the value of the exponent is close to $n / 2$, i.e. the following holds.

Theorem 1. $s(n)=2^{n / 2+o(n)}$.

In this note we study the behaviour of $s_{\max }(n)$. Cameron and Erdös [4] observed that $s_{\max }(n) \geq 2^{\lfloor n / 4\rfloor}$ and asked if $s_{\max }(n)=o(s(n))$, or, maybe, even $s_{\max }(n) \leq$ $2^{n / 2-\epsilon n}$, holds for some constant $\epsilon>0$. Our main result states that this is indeed the case.

Theorem 2. There exists $n_{0}$ such that for $n \geq n_{0}$ we have $s_{\max }(n) \leq 2^{n / 2-2^{-28} n}$.

For a maximal sum-free set $A \subseteq[n]$ and $B \subseteq A$ let

$h_{A}(B)=|[n] \backslash[(A \backslash B) \cup((A \backslash B)+(A \backslash B)) \cup((A \backslash B)-(A \backslash B)) \cup(A \backslash B) / 2]|$, i.e. $h_{A}(B)$ denotes the number of elements $i \in[n]$ one can add to $A \backslash B$ so that $\{i\} \cup(A \backslash B)$ remains sum-free. Our argument relies on the following result of probabilistic flavor.

Lemma 3. Let $\beta$ be a constant such that $0<\beta<1 / 2$. Then there exists $n_{0}$ such that for $n \geq n_{0}$, every maximal sum-free set $A \subseteq[n]$ with $|A|=m \geq n / 11$ contains at least $n^{-5 \sqrt{n}}\left(\begin{array}{c}m \\ k\end{array}\right)$ subsets $B$ of $k=\lfloor\beta m\rfloor$ elements for which $h_{A}(B) \leq 2 \beta n$.

Proof. For each $i \in[n] \backslash A$ let us choose a pair $R_{i}=\left\{a_{i}^{\prime}, a_{i}^{\prime \prime}\right\}$ of two, not necessarily distinct, elements of $A$ such that either $i=a_{i}^{\prime}+a_{i}^{\prime \prime}$, or $i=a_{i}^{\prime}-a_{i}^{\prime \prime}$, or, maybe, $2 i=a_{i}^{\prime}=a_{i}^{\prime \prime}$ (since $A$ is a maximal sum-free set such a pair always exists). Let

Received by the editors September 7, 1999 and, in revised form, December 13, 1999.

2000 Mathematics Subject Classification. Primary 11B75; Secondary 05A16.

The first author was supported in part by KBN Grant 2 P03A 02117. 
$A^{\prime}$ be a set of all $a \in A$ which belong to at most $\sqrt{n}$ from the sets $R_{i}, i \in[n] \backslash A$. Clearly, $m^{\prime}=\left|A^{\prime}\right| \geq|A|-2 \sqrt{n}$. Let

$$
W=\left\{i: R_{i} \cap A^{\prime} \neq \emptyset\right\},
$$

and denote by $\mathbf{B}$ a set chosen uniformly at random among all subsets of $A^{\prime}$ with $k$ elements. We shall study the behaviour of the random variable $\mathbf{X}=\sum_{i \in W} \mathbf{X}_{i}$, where for $i \in W$

$$
\mathbf{X}_{i}= \begin{cases}0 & \text { if } R_{i} \cap \mathbf{B}=\emptyset, \\ 1 & \text { if } R_{i} \cap \mathbf{B} \neq \emptyset .\end{cases}
$$

For the expectation of $\mathbf{X}$, we get

$$
\begin{aligned}
\mathrm{EX} & =\sum_{i \in W} \mathrm{E} \mathbf{X}_{i} \leq|W|\left(1-\min \left\{\frac{\left(\begin{array}{c}
m^{\prime}-2 \\
k
\end{array}\right)}{\left(\begin{array}{c}
m^{\prime} \\
k
\end{array}\right)}, \frac{\left(\begin{array}{c}
m^{\prime}-1 \\
k
\end{array}\right)}{\left(\begin{array}{c}
m^{\prime} \\
k
\end{array}\right)}\right\}\right) \\
& \leq(1+o(1))(n-m)\left(1-\left(1-\left(k / m^{\prime}\right)^{2}\right)\right) \leq 2 \beta n-2 \beta m .
\end{aligned}
$$

In order to estimate the variance $\operatorname{Var} \mathbf{X}$ note that

$$
\operatorname{Var} \mathbf{X} \leq \sum_{i, j: R_{i} \cap R_{j} \neq \emptyset}\left|\operatorname{Cov}\left(\mathbf{X}_{i}, \mathbf{X}_{j}\right)\right|+\sum_{i, j: R_{i} \cap R_{j}=\emptyset}\left|\operatorname{Cov}\left(\mathbf{X}_{i}, \mathbf{X}_{j}\right)\right| .
$$

As a crude upper bound for the first sum one can take

$$
\sum_{i, j: R_{i} \cap R_{j} \neq \emptyset}\left|\operatorname{Cov}\left(\mathbf{X}_{i}, \mathbf{X}_{j}\right)\right| \leq \sum_{i, j: R_{i} \cap R_{j} \neq \emptyset} 1 \leq 2 n \sqrt{n},
$$

while the second sum is not larger than

$$
\left(\sum_{i \in W} \mathrm{E}_{i}\right) m^{\prime}\left[\left|\frac{\left(\begin{array}{c}
m^{\prime}-2 \\
k
\end{array}\right)}{\left(\begin{array}{c}
m^{\prime} \\
k
\end{array}\right)}-\frac{\left(\begin{array}{c}
m^{\prime}-4 \\
k-2
\end{array}\right)}{\left(\begin{array}{c}
m^{\prime}-2 \\
k-2
\end{array}\right)}\right|+\left|\frac{\left(\begin{array}{c}
m^{\prime}-1 \\
k
\end{array}\right)}{\left(\begin{array}{c}
m^{\prime} \\
k
\end{array}\right)}-\frac{\left(\begin{array}{c}
m^{\prime}-3 \\
k-1
\end{array}\right)}{\left(\begin{array}{c}
m^{\prime}-1 \\
k-1
\end{array}\right)}\right|\right] \leq \mathrm{E} \mathbf{X} m^{\prime} \frac{11}{m^{\prime}} \leq 11 \mathrm{E} \mathbf{X}
$$

Hence

$$
\operatorname{Var} \mathbf{X} \leq 11 \mathrm{E} \mathbf{X}+2 n \sqrt{n} \leq 3 n \sqrt{n}=o\left(n^{2}\right),
$$

and so Chebyshev's inequality implies that, for $n$ large enough, with probability at least $1 / 2$ we have $\mathbf{X} \leq 2 \beta n-\beta m$. Thus, since $h_{A}(\mathbf{B}) \leq \mathbf{X}+|\mathbf{B}|$, there exist at least

$$
\frac{1}{2}\left(\begin{array}{c}
m^{\prime} \\
k
\end{array}\right) \geq n^{-5 \sqrt{n}}\left(\begin{array}{c}
m \\
k
\end{array}\right)
$$

subsets $B$ of $A$ with $k$ elements for which $h_{A}(B) \leq 2 \beta n$.

Proof of Theorem Q Let us first estimate the number $s_{\max }^{\prime}(n)$ of all maximal sumfree subsets of $[n]$ with at least $n / 11$ elements. Set $\beta=2^{-23}$ and consider the number $t$ of all pairs $(A, A \backslash B)$, where $A$ is a maximal sum-free set of at least $n / 11$ elements and $B \subseteq A$ is such that $|B|=\lfloor\beta|A|\rfloor$ and $h_{A}(B) \leq 2 \beta n$. Then, due to Lemma 3, for $n$ large enough we have

$$
t \geq s_{\max }^{\prime}(n) n^{-5 \sqrt{n}}\left(\begin{array}{l}
|A| \\
|B|
\end{array}\right) \geq s_{\max }^{\prime}(n) n^{-5 \sqrt{n}}\left(\begin{array}{c}
\lfloor n / 11\rfloor \\
\lfloor\beta n / 11\rfloor
\end{array}\right) \geq s_{\max }^{\prime}(n) \beta^{-\beta n / 11} .
$$


On the other hand, there are at most $2^{2 \beta n}$ ways to enlarge a set $A \backslash B$ to a maximal sum-free set. Thus, $t \leq s(n) 2^{2 \beta n}$ and using Theorem 1 we infer that

$$
s_{\max }^{\prime}(n) \leq s(n) 2^{2 \beta n} \beta^{\beta n / 11} \leq 2^{n / 2+o(n)}\left(4^{11} \beta\right)^{\beta n / 11} \leq 2^{n / 2-2^{-27} n} .
$$

Hence

$$
s_{\max }(n) \leq s_{\max }^{\prime}(n)+\sum_{i=1}^{n / 11}\left(\begin{array}{c}
n \\
i
\end{array}\right) \leq 2^{n / 2-2^{-27} n}+2^{49 n / 100} \leq 2^{n / 2-2^{-28} n} .
$$

Note that in the proof of Theorem 2 we used no arithmetic properties of the natural numbers. Thus, our result can be generalized as follows. Let $\oplus: \Omega \times \Omega \rightarrow \Omega$ be an operation in $A$. We say that $A \subseteq \Omega$ is $\oplus$-free if $a \oplus b \notin A$ for $a, b \in A$ and define a maximal $\oplus$-free set accordingly. Furthermore, for $A \subseteq \Omega$, let $\sigma(A)$ and $\sigma_{\max }(A)$ denote the number of $\oplus$-free and maximal $\oplus$-free subsets of $A$, respectively. Then one can mimic our argument to show the following result.

Theorem 4. For every $\alpha>0$ there exist $\epsilon>0$ and $n_{0}$ such that the following holds. For every set $\Omega$ with operation $\oplus: \Omega \times \Omega \rightarrow \Omega$ and every $A \subseteq \Omega$ for which $|A|=n>n_{0}$ and $\sigma(A) \geq 2^{\alpha n}$, we have $\sigma_{\max }(A) \leq \sigma(A) 2^{-\epsilon n}$.

Finally, we remark that Cameron [3] studied the set of all subsets of the natural numbers as a metric space with the distance defined as

$$
d(A, B)=2^{-\min \{A \triangle B\}},
$$

where $A \triangle B$ denote the symmetric difference of sets $A$ and $B$. Then the set of all sum-free subsets of natural numbers has Hausdorff dimension $1 / 2$ (it follows immediately from Theorem 1 and the fact that all sets which contain only odd numbers are sum-free). Theorem 2 implies that the dimension of the set of all maximal sum-free subsets of the natural numbers is at most $1 / 2-2^{-28}<1 / 2$.

\section{REFERENCES}

1. N. Alon, Independent sets in regular graphs and sum-free subsets of finite groups, Israel J. Math 73 (1991), 247-256. MR 92k:11024

2. N. Calkin, On the number of sum-free sets, Bull. London Math. Soc. 22 (1990) 141-144. MR 91b:11015

3. P. Cameron, Portrait of a typical sum-free set. In "Survey in Combinatorics 1987" (C. Whitehead, ed.), London Mathematical Lecture Note Ser. 123, Cambridge University Press, 1987, 13-42. MR 88k:05138

4. P. Cameron and P. Erdös, On the number of sets of integers with various properties. In "Number Theory: Proc. First Conf. Can. Number Th. Ass." (R.A. Mollin, ed.), Banff, 1988, de Gruyter, 1990, 61-79. MR 92g:11010

Department of Discrete Mathematics, Adam Mickiewicz University, ul. Matejki 48/49, 60-769 Poznań, Poland

E-mail address: tomasz@amu.edu.pl

Mathematisches Seminar, Universität Zu Kiel, Ludewig-Meyn-Str. 4, 24098 Kiel, Germany - Department of Discrete Mathematics, Adam Mickiewicz University, ul. Matejki 48/49, 60-769 Poznań, Poland

E-mail address: tos@numerik.uni-kiel.de 